\title{
Safe and Efficient Human-Robot Collaboration Part I: Estimation of Human Arm Motions
}

\author{
Roman Weitschat, Jan Ehrensperger, Moritz Maier, and Harald Aschemann
}

\begin{abstract}
A significant barrier regarding a successful implementation of fenceless robot cells into manufacturing areas with humans is given by the inefficiency due to safety requirements. Robot motions have to be slowed down so that an unexpected collision with a human does not result in human injuries. This velocity reduction leads to longer cycle times and, hence, fenceless robot cells turn out as uneconomic. In this paper, a new approach for human-robot collaboration in assembly tasks is presented. For a better performance of the robot, methods are investigated on how the robot can exploit a maximum performance while maintaining the safety of collaborating humans. For this purpose, the kinematics and dynamics of a human arm are described by a control-oriented dynamic model to determine its capability and reachability. Successful experiments validate the dynamic model as well as a corresponding projection approach for calculating possible movements of the human arm that may lead to a collision with the robot. Finally, this information is used to calculate an admissible path velocity that minimizes the danger of human injuries.
\end{abstract}

\section{INTRODUCTION}

Human-robot collaboration (HRC) as exemplary shown in Fig. 1 is a very important topic in industrial robotics [1], especially the field of human safety. Asimov's first law: "A robot may not harm a human being" must be strictly satisfied. Therefore, the International Standards Organisation (ISO) has defined standards or requirements that should be met by companies using work cells with HRC. The standard ISO 10218 defines several requirements like safetyrated monitored stop, hand guiding, speed and separation monitoring, and power and force limiting by inherent design. The latter was extended with the ISO/TS 15066, which considers the transient and quasi-static impact force as well as pressure on the human skin. To comply with these limits, robotic companies have developed many collaborative robots, for example, KUKA iiwa, UR 5 and Franka. These robots enable external torques to be measured, which trigger an emergency stop when the thresholds are exceeded. To prevent from any injuries, they also avoid sharp edges at the robot structure.

Even with these collision detection mechanisms in place, the velocity of the robot has to be reduced significantly, such that the ISO requirements are satisfied [2]. The underlying motivation for this restriction is that a collision could happen at any time. In this paper, we convert this assertion to a

Roman Weitschat is with the Robotics and Mechatronics Center, DLR - German Aerospace Center, Wessling, Germany, roman. weitschat@dlr.de

Prof. Dr. Harald Aschemann is with the Chair of Mechatronics, University of Rostock, Germany harald.aschemanneuni-rotock.de

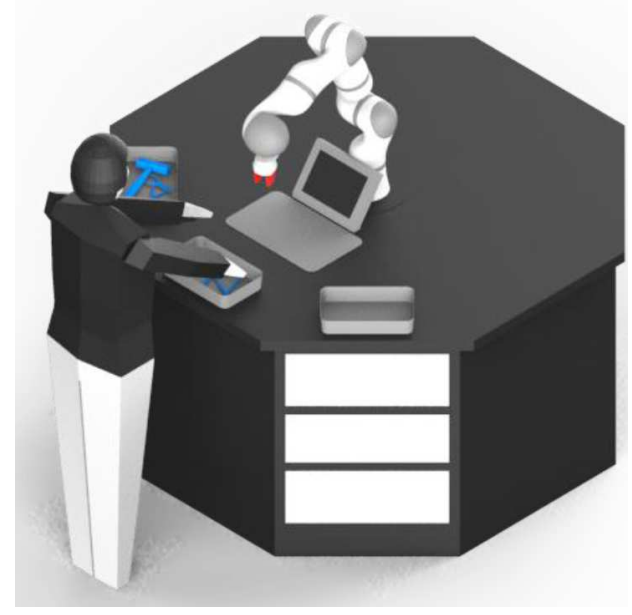

Fig. 1. Schematic view of industrial human-robot collaboration in a assembly process where robots are assisting shop floor workers - they take over complex manufacturing tasks to improve the productivity in combination with a flexibility due to human support.

question: "Will a collision with a human occur if I execute this trajectory?" Cages around industrial robots ensure that the answer is always 'no', and velocities must therefore not be constrained for human safety reason. For 'static' humans, i.e., humans that do not move, this question is also easily answered: if the human is in my trajectory 'yes', and if he is not 'no'.

For humans who move - as is our nature - the answer to this question depends on two factors. The first is the intention of the human. We assume the worst-case scenario, where a human intentionally causes a collision with the moving robot. Usually, humans do not deliberately cause collisions, but may still display equivalent behavior when not observing the entire situation. As we want to guarantee that harmful collisions are avoided, we must assume the worst possible behavior from the human, which is an intentional collision. The second factor is human dynamics. Even if a human would want to cause a collision with all its might, a collision may be physically impossible due to kinematic and dynamic constraints of the human. For instance, the trajectory of the robot may simply be out of range, or the inertia of the arm may prevent the human from being fast enough to move the arm within the trajectory of the robot, as shown in Fig.2.

The core idea behind this paper is to provide the robot with a real-time kinematic and dynamic model of the human arm. Thereby, a prediction becomes possible whether a human would be able to cross the trajectory of the robot. The worstcase assumption is that the intention of the human is to do so 
Case 1

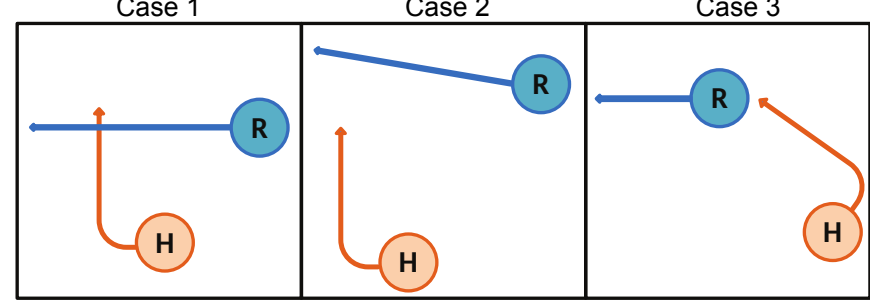

Fig. 2. Differentiation between three cases: Case 1: The human is fast enough to cross the robot path. Case 2: The kinematic limits of the human are reached. Case 3: The robot is able to move very fast, the human cannot cross the robot path.

(see Fig.3). If collisions are thus predicted to be impossible, robots are able to move on faster velocities, even in the presence of moving humans. Our ultimate aim is thus to improve the performance of robots, whilst respecting the ISO requirements on safety.

The paper is organized as follows: First, the related work is discussed and human arm motion experiments are presented where humans have to slap down a softball from different positions as fast as possible. Furthermore, the innovative approach for a safe and effective collaboration of humans and robots is introduced, addressing the kinematics and dynamics of the human arm model. Furthermore, the human arm state observer (HASO) will be presented and the calculation of the projection is explained, which is then evaluated with human arm motion experiments. Finally, conclusions are provided.

\section{RELATED WORK}

In scientific publications, injuries caused by a collision of a human with a robot are treated with a focus on the parameters having an impact on the severity of injury [3], [4].

Three general influencing factors are known to be important:

- Geometry - sharp impactor, blunt impactor

- Mass of the impactor

- Velocity of the impactor

Usually, the velocity of the manipulating robot is reduced to a safe motion, because changing the mass and geometry of a carried object is unfeasible. In real assembly tasks, however, reducing the velocity results in a very slow execution of the working steps. The use of a robot in an interactive task aims at creating an added value to the work process. A common speed reduction often leads to the point where any advantage has gone lost.

In these considerations, the human is treated as static, and the behavior of the human is not taken into account. In the future, robots should avoid collisions with humans during their motion. Therefore, many researchers are working on a collision-avoiding path planning. Three-dimensional collision avoidance was proposed in [5] or approaches based on potential fields for collision avoidance in real-time [6], [7]. New sensor technologies allow for tracking human positions, for example, vision-based human tracker or tracking markers from the VICON.

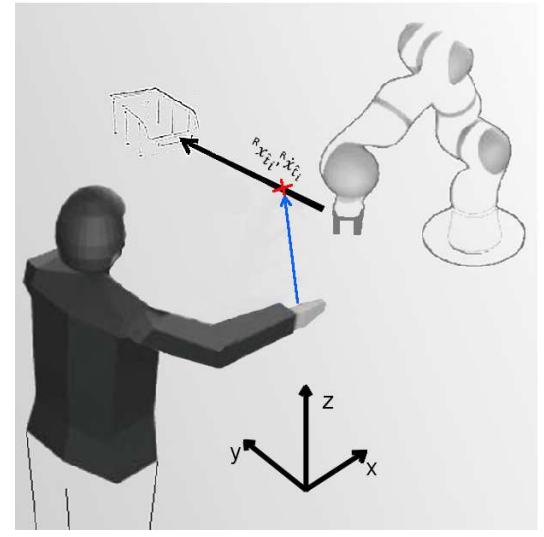

Fig. 3. Human arm motion projection into the direction of the desired robot path. Calculation of the time up to a possible collision between the robot and the human arm by using a low-order dynamical arm model accounting for limited torques.

Given this sensor technology, new approaches emerged in anticipating the human behavior, where the goal of the human activities is estimated and the robot is able to react [8]. In [9], the authors predict human motions from learned human movements and estimate the resulting path of a human. The result is given by the most probable human motion or the probability of a human goal. Another approach evaluating the human and the robot motion with a possible interception was discussed in [10], where a prediction of pedestrian movements was presented. Also mobile robots predict the motion of humans in relation to its path and calculate an alternative part for the robot motion, if necessary, see [11], [12], [13]. One issue with these approaches is that they employ only purely kinematic models which result in an inaccurate and imperfect representation of the human motion.

In the following, human arm motion experiments are presented to map real motions into the proposed projection scheme.

\section{HUMAN ARM MOTION EXPERIMENTS}

In a first step, realistic human arm motions are measured using a VICON tracking system. The scenario of the experiments corresponds to a worker sitting in front of an assembly workbench during his working hours. The person is located in front of the table in a sitting position, with his right hand in a fixed resting position. Tracking markers are attached to the human arm to measure necessary limb positions. One constraint during the experiments is to suppress the motion of the upper body. Thereby, the complexity is kept as low as possible. Due to the fact that the human body position changes in real situations, the shoulder position is subtracted from the final values.

\section{A. Experimental setup}

The experimental setup is structured as follows: The human is sitting in front of a table, which represents a common situation in an industrial manufacturing. VICON markers are placed on the human to determine the position of each arm joint. The first marker is placed at the back of the hand, the second at the elbow and the third at the 


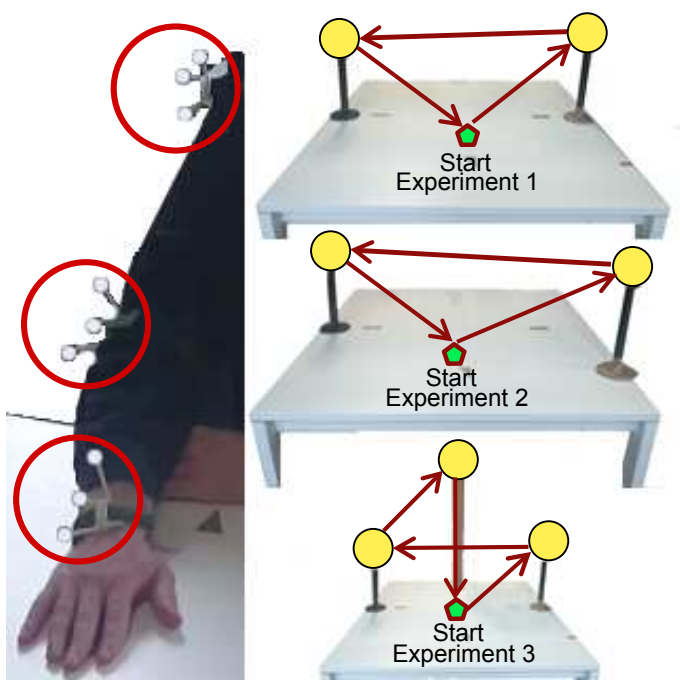

Fig. 4. In these experiments the human experimenters should throw the objects from the pillars as fast as possible. Starting in a rest position the human arm has to be accelerated to be as fast as possible

shoulder, as shown in Fig. 4, left side. The marker positions are recorded with a frequency of $100 \mathrm{~Hz}$.

The task is designed as follows: In front of the human, two or three pillars were placed with a foam ball on it, as depicted in Fig. 4, right row. Three different motions are executed to obtain an enhanced representation of the human arm motion for further considerations in the projection. The task for the human experimenter was to slap down a softball from each of these pillars as fast as possible. Experiment one includes two pillars which have the same distance to the subject with the positions $P_{1}=[0.4 ; 0.20 .15] \quad$ and $P_{2}=[0.4-0.20 .15]$. The height is chosen to be in a usual height a robot would execute the motion. In the second experiment, the first pillar is moved closer to the human with $P_{1}=\left[\begin{array}{lll}0.2 & 0.2 & 0.15\end{array}\right]$ and $P_{2}=[0.4-0.20 .15]$. In the last experiment, an additional pillar is placed at $P_{1}=\left[\begin{array}{lll}0.4 & 0.2 & 0.15\end{array}\right]$, $P_{2}=\left[\begin{array}{lll}0.4-0.2 & 0.15\end{array}\right]$ and $P_{3}=\left[\begin{array}{lll}0.5 & 0.0 & 0.45\end{array}\right]$ to obtain measurements at the kinematic limits of a human arm, too.

\section{B. Experiment results}

In an experiment with a human, as described above, the collected data was analyzed. In Fig. 5, the motions of the human hand are illustrated in Cartesian space. In Experiment 1 shown in Fig. 5(a), the time necessary to hit the first position if the first pillar is approximately $60 \mathrm{~ms}$, which is depicted as colored circle with a scale from red to green. The motion by $0.266 \mathrm{~m}$ in $0.06 \mathrm{~s}$ results in an average velocity of $4.43 \mathrm{~m} / \mathrm{s}$. The turning process at position $P=\left[\begin{array}{lll}0.2 & 0.2 & 0.2\end{array}\right]$ including the deceleration and acceleration requires around $20 \mathrm{~ms}$, which is due to the inertia of the human arm. The motion time to position two is $60 \mathrm{~ms}$ for a distance of $0.4 \mathrm{~m}$. This dynamical behavior is used to determine the parameters of the human arm model in Sec.IV-A. The results in experiment 2 and 3 indicate a comparable dynamic behavior of the human arm motion. In the following section, these experimental results are used to

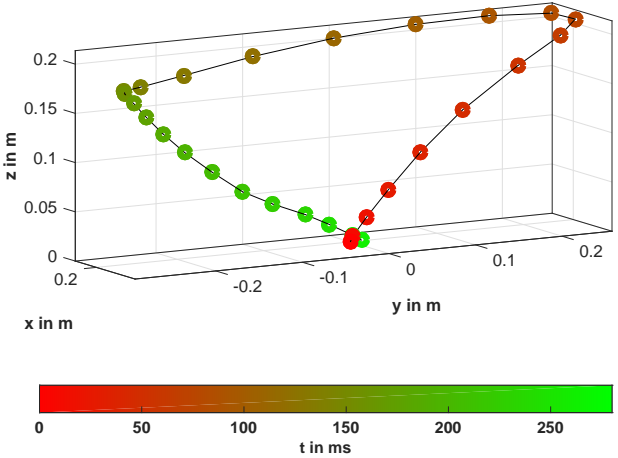

(a) Experiment 1

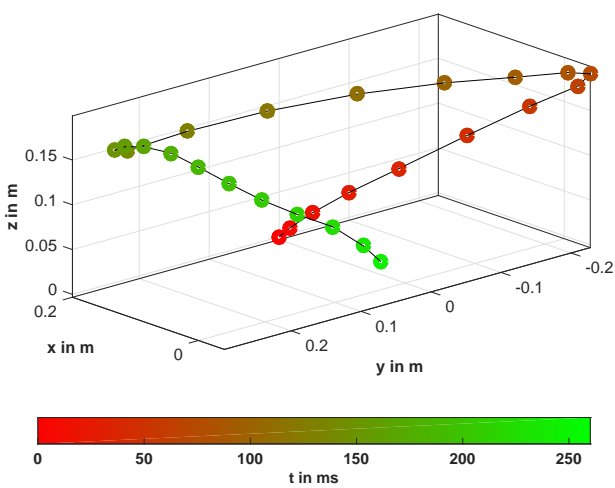

(b) Experiment 2

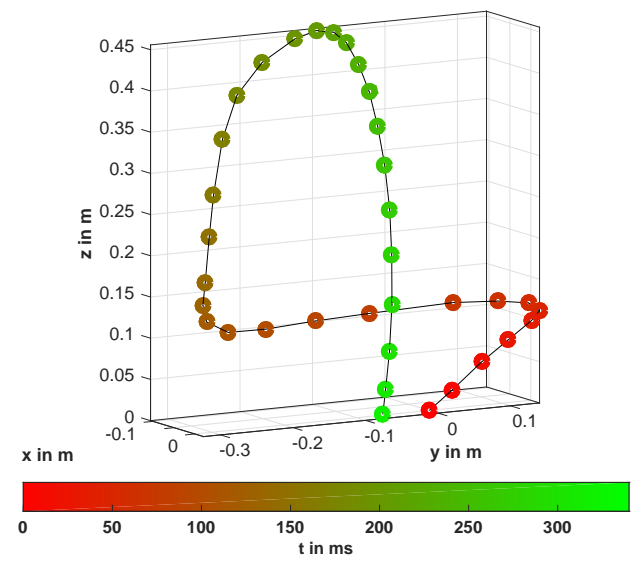

(c) Experiment 3 full

Fig. 5. Measurements of the human motion from the experiments described in Fig.4

validate the developed algorithm to project human motions into the path of the robot.

\section{HUMAN ARM MOTION DETECTION AND ESTIMATION}

In this section, the dynamic model of the human arm is described. Then, the structure of the calculation steps is explained: measurements of the human arm position serve as input for an observer-like structure that calculates the estimated joint values, which are used within the motion simulator of the human arm as illustrated in Fig.7, left row. In this paper, however, we consider only the position of the hand; this approach prevents from placing markers all over the arm. The current estimated joint values - e.g., 
joint positions $\hat{q}$ and velocities $\hat{\dot{q}}$ - are used as inputs of the projection algorithm, depicted in Fig. 7 right side. The calculated time $\tilde{t}$ is required by the human arm to attain the robot path which finally enables the maximum value of the allowed robot velocity.

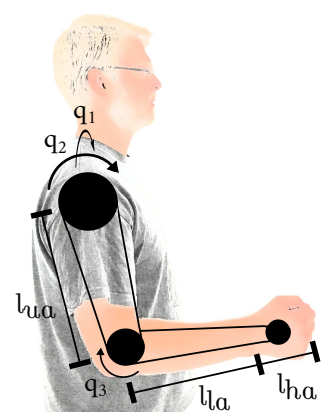

Fig. 6. Kinematic description of the simplified human arm model. The length of the arm links are defined by $l_{u a}, l_{l a}$, and $l_{h a}$. The rotation of the arm joints are described by $q_{1}, q_{2}$, and $q_{3}$

\section{A. Simplified human arm model}

In the robotics literature, usually the human arm is described as a planar two DoF system to obtain the kinematics as well as the dynamics of human arm motions, see [14], [15]. The requirements regarding the dynamic model are:

1) The human arm model should allow for an arbitrary position in the three-dimensional Cartesian space. The orientation of the human hand, however, is not of interest in the given case.

2) The dynamic model is real-time capable, where the computation time should be as low as possible.

As a consequence, a three DoF human arm model has been chosen. A real-time simulation of a three DoF arm model was shown in [16], where the focus was on using the performance for electrical stimulation. The given ambiguity - e.g., the possibility to move the elbow while the hand position is almost constant - was not taken into account. With only the measured human hand positions available, a calculation of the real elbow position is not possible. Obviously, this represents a direction of future research and a further step to get closer to a more precise simulation of the human arm motion.

The human body is treated as a fixed base for the human arm, which is related to the envisaged complexity reduction in this paper. The base coordinate system is located in the shoulder joint. The generalized coordinates are defined as shown in Fig. 6 A positive shoulder rotation by $q_{1}$ describes the motion to the right side of the human. The rotation by $q_{2}$ leads to a motion to the back of the human and the elbow rotation, characterized by $q_{3}$. With these three DoF, the work space in the Cartesian space is identical to the one of a real human arm. In order to have a close representation to a human arm, values for human arm dimensions could be taken from [17]. In this paper, however, the measured arm dimensions of the experimenter are employed to specify the human arm model: The upper arm has a length of $l_{u a}=290 \mathrm{~mm}$, whereas the lower arm is characterized

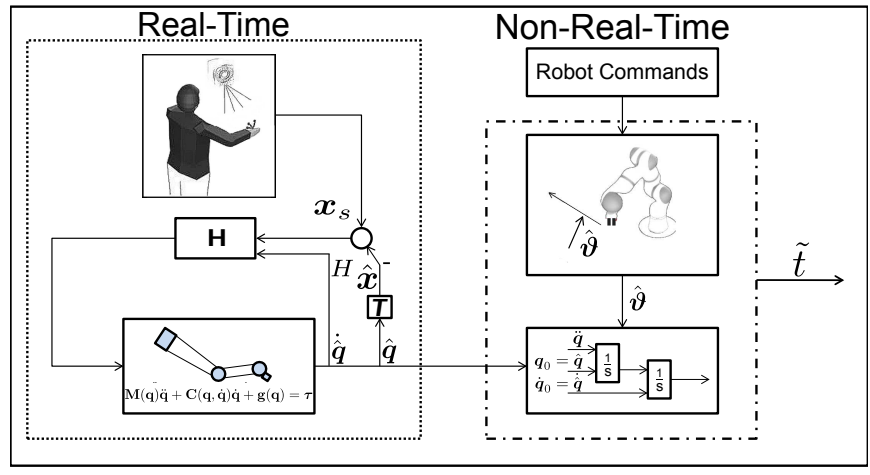

Fig. 7. Structure of predicted human motion into the direction of the robot path. Calculation of the necessary time to cross the robot path given the dynamics and limited torques of the human arm. The predicted time to attain the robot path - if possible - affects the admissible velocity profile of the robot.

by $l_{l a}=250 \mathrm{~mm}$ and the hand by $l_{h a}=190 \mathrm{~mm}$. For simplicity, the hand and lower arm are treated as rigid unit. For the human arm model with two links, the final length of the lower arm is given by $0.416 \mathrm{~mm}$. Typical joint limitations of the human arm can be found in [18].

The equations of motion for a human arm are described by

$$
\mathbf{M}(\mathbf{q}) \ddot{\mathbf{q}}+\mathbf{C}(\mathbf{q}, \dot{\mathbf{q}}) \dot{\mathbf{q}}+\mathbf{g}(\mathbf{q})=\boldsymbol{\tau}
$$

with the symmetric and positive definite mass matrix $\mathbf{M}(\mathbf{q}) \in \Re^{3 x 3}$, the centrifugal and Coriolis torques $\mathbf{C}(\mathbf{q}, \dot{\mathbf{q}}) \in \Re^{3 x 1}$ and the gravity torques $\mathbf{g} \in \Re^{3 x 1}$, corresponding to a typical $3-\mathrm{DoF}$ pendulum. The system input is given by the torque vector $\tau \in \Re^{3 x 1}$. Values for the mass, the center of mass and the resulting inertia of the human arm model correspond to the values given in [17], where the center of mass is located in the middle of the links. The arm segments are approximated by rigid rods for the upper arm and the lower arm and combined with the hand. The three DoF dynamic arm model is an appropriate representation of the real human arm. The reproduction of a precise model of the human arm including muscle model would significantly increase the complexity of the simulation. Therefore, the mapping on a non-complex three DoF system leads to the lowest computing time for a dynamic model in the three dimensional Cartesian space. As the position of the elbow is not measured, a representation of the elbow is omitted. This is a consequence of using just one marker and the measurement of the human hand position only. The absence of the representation of the elbow leads to the necessity of the HASO to observe current joint angles, which is presented in the next section.

\section{B. Human arm state observer}

The input to the HASO are the measured Cartesian positions of the human arm. This could be either done by a marker-based tracking system like a VICON or a visionbased human tracker like a Microsoft Kinect. In the given case, the usage of a VICON system is preferred due to a higher measurement frequency and more accurate position measurements. 

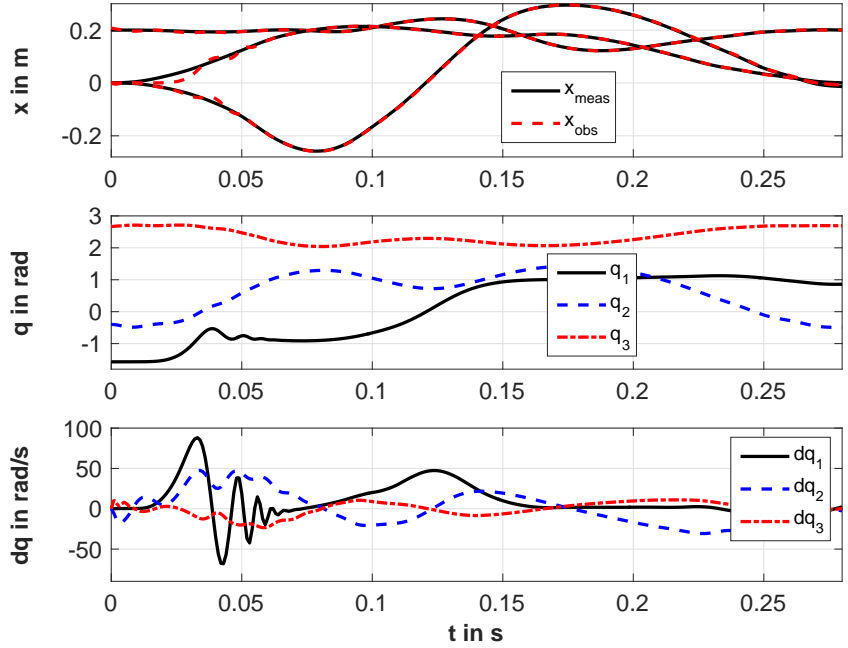

Fig. 8. Estimates of the joint angles as well as velocities provided by the HASO

As shown in Fig. 7, $\mathbf{x}_{\mathbf{s}}$ defines the measured Cartesian position of the human hand. These measured position values are employed as inputs of a impedance-based feedback controlled system that provides the current velocity of the joint coordinates. For this purpose, the transformation from work space coordinates to joint angles is needed. The equations of motion for the HASO are given by

$$
\begin{aligned}
{ }^{H} \dot{\hat{\mathbf{q}}}_{t} & =\mathbf{f}\left({ }^{H} \hat{\mathbf{q}}_{t}, \mathbf{u}_{t}\right)+\mathbf{H}\left(\mathbf{x}_{\mathbf{s}},{ }^{H} \hat{\mathbf{x}}_{t},{ }^{H} \hat{\mathbf{q}}_{t}\right), \\
{ }^{H} \hat{\mathbf{x}}_{t} & =\mathbf{T}\left({ }^{H} \hat{\mathbf{q}}_{t}\right),
\end{aligned}
$$

where ${ }^{H} \hat{\mathbf{q}}_{t}=\left[{ }^{H} \hat{\mathbf{q}}_{t}{ }^{H} \dot{\hat{\mathbf{q}}}_{t}\right]^{T},{ }^{H} \hat{\mathbf{q}}_{t} \in \Re^{3}$ denotes the estimated generalized coordinates in joint space of the low-order dynamical arm model, ${ }^{H} \mathbf{x} \in \Re^{3}$ the Cartesian coordinates of the hand position, and $\mathbf{u}_{t} \in \Re^{3}, \mathbf{u}_{t}=\mathbf{0}$, the control input to the dynamic system. The vector field $\mathbf{f}$ describes the reformulated non-linear differential equation from (1) with respect to ${ }^{H} \hat{\mathbf{q}}_{t}$, and $\mathbf{T}$ is the transformation from joint space into Cartesian space. Unfortunately, the input is not measurable yet in the given case 1 . The control torques within the HASO are given by [19]

$$
\mathbf{H}=\mathbf{g}_{q}\left({ }^{H} \hat{\mathbf{q}}_{t}\right)-\left(\frac{\partial \mathbf{V}_{i m p}\left(\mathbf{x}_{\mathbf{s}},{ }^{H} \hat{\mathbf{q}}_{t}\right)}{\partial^{H} \hat{\mathbf{q}}_{t}}\right)^{T}-\mathbf{D}_{i m p}\left({ }^{H} \hat{\mathbf{q}}_{t}\right)^{H} \dot{\hat{\mathbf{q}}}_{t},
$$

where $\mathbf{g}_{q}\left({ }^{H} \mathbf{q}_{t}\right)$ describes the gravity compensation, whereas the impedance control involves a spatial spring - related to the potential $\mathbf{V}_{i m p}$ - as well as a positive definite matrix $\mathbf{D}_{i m p}$, which is realized by eigenvalue decomposition. The VICON system provides a six-dimensional position and orientation vector $\mathbf{x}_{s}$, where the Cartesian position serves as the input for the HASO. The results of the HASO are presented in Fig.8. The tracking behavior is shown in the upper plot. The both lower plots depict the mapping into joint coordinates for the reduced dynamic human arm model.

\footnotetext{
${ }^{1}$ Torque measurements in the human arm can be realized, for example, by surface electromyography (sEMG) sensors, which could be subject of future work.
}

\section{Human arm motion projection}

For the human arm projection, the dynamical model described in Eq. (1) is used to predict the human arm motion towards the direction of the robot path. The current human arm position $\mathbf{x}_{s}$ is measured by the VICON, whereas the current joint states are calculated. They serve as initial inputs to the following human arm motion projection algorithm. The current position of the robot is measured internally by the joint sensors. A transformation into the Cartesian work space results in the robot end-effector position ${ }^{R} \mathbf{x}_{0}$. With the maximum Cartesian speed derived from the measurements and the estimated hand position, the approximate time span $\hat{t}$ can be calculated until a collision may occur. The current robot velocity is given by ${ }^{R} \dot{\mathbf{x}}_{0}$.

For the control of the closed-loop simulation, a simple impedance controller using $\boldsymbol{\tau}=\mathbf{J}^{T} \mathbf{F}$ as described in [20] is not suitable because the human arm attains a very high speed. The selected stiffness as implemented in the observer is very large and does not allow for an adequate representation. This is caused by the closed-loop behaviour of the HASO. As a consequence of the robot configuration and the low number of DoF, low stiffness may lead to large local path errors. Therefore, the implementation of an admittance control seems beneficial to approximate the motion of a human arm. The direction vector is given by

$$
\hat{\boldsymbol{\vartheta}}=\frac{{ }^{R} \boldsymbol{x}_{\hat{t}_{i}}-\mathbf{x}_{s}}{\left\|{ }^{R} \boldsymbol{x}_{\hat{t}_{i}}-\mathbf{x}_{s}\right\|}
$$

where ${ }^{R} \boldsymbol{x}_{\hat{t}_{i}}$ denotes the approximated robot position at an estimated collision time $\hat{t}_{i}$. This point can be achieved by approximating the motion of the human and the robot. The human needs ${ }^{H} \tilde{t}=0.08 \mathrm{~s}$ for the relative motion of ${ }^{H} \tilde{x}=0.4 \mathrm{~m}$ obtained from the experiments which leads to an approximated time constant of $t^{*}=\frac{{ }^{H} \tilde{t}}{{ }_{\tilde{x}}}=0.2 \frac{\mathrm{s}}{\mathrm{m}}$. The approximated collision position can be obtained with

$$
{ }^{R} \boldsymbol{x}_{\hat{t}_{i}}={ }^{R} \boldsymbol{x}_{t_{0}}+t^{*}\left({ }^{R} \boldsymbol{x}_{t_{0}}-\mathbf{x}_{s}\right){ }^{R} \dot{\boldsymbol{x}}_{t_{0}}
$$

where ${ }^{R} \boldsymbol{x}_{t_{0}}$ denotes the current robot position. The approximated time to collision is described by $\hat{t}_{i}=t^{*}\left({ }^{R} \boldsymbol{x}_{t_{0}}-\mathbf{x}_{s}\right)$, and ${ }^{R} \dot{\boldsymbol{x}}_{t_{0}}$ represents the current robot velocity. For the desired joint values of the projection, the admittance is defined as

$$
{ }^{H} \boldsymbol{q}_{d e s}=\int \boldsymbol{J}_{d}^{*}(\kappa \hat{\boldsymbol{\vartheta}}-\eta \dot{\boldsymbol{x}}) d t
$$

where $\boldsymbol{J}_{d}^{*}$ denotes the regularized pseudo-inverse jacobian matrix with $\boldsymbol{J}_{d}^{*}=\boldsymbol{J}^{T}\left(\boldsymbol{J} \boldsymbol{J}^{T}+\lambda^{2} \boldsymbol{I}\right)^{-1}$, with $\lambda$ as a regularization parameter [21]. The parameter $\kappa$ and $\eta$ are gains for the direction vector as well as the Cartesian damping term with the Cartesian velocity $\dot{\boldsymbol{x}}$. The desired joint values are generated by the admittance control and serve as inputs for underlying standard PID controller with limited gains for a high accuracy with respect to the dynamic projection. The resulting time $\tilde{t}$, obtained from the simulation with (1) and the initial values from (2), a human needs to reach the robot 

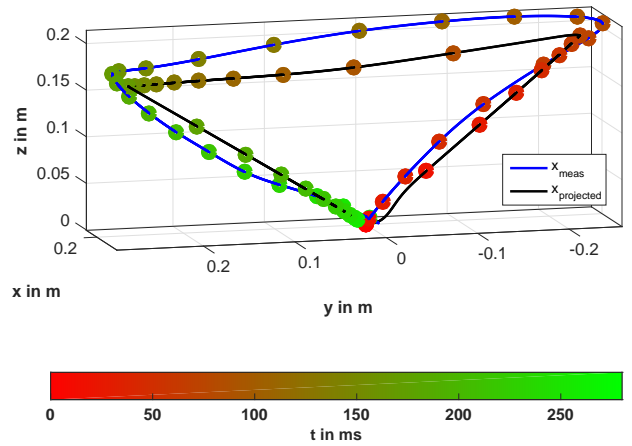

(a) Projection of the motion from experiment 1

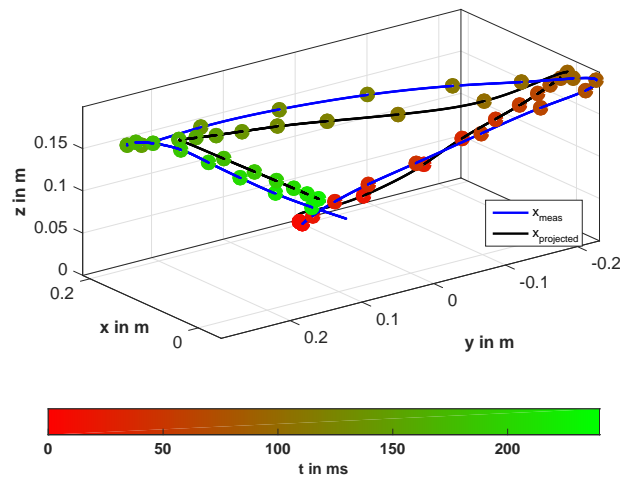

(b) Projection of the motion from experiment 2
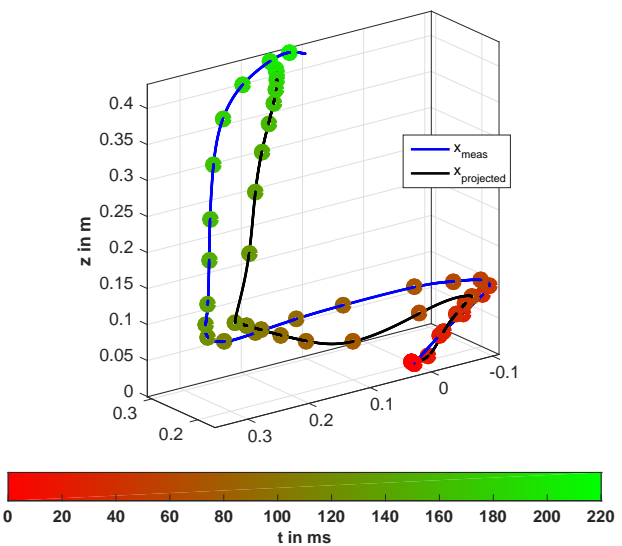

(c) Projection of the motion from experiment 3

Fig. 9. Comparison of projected motions of the human model with the measurements from the experiments described in Fig. 4.

path is used to determine the resulting maximum velocity of the robot

$$
{ }^{R} \dot{\boldsymbol{x}}_{\text {max }}={ }^{R} \dot{\boldsymbol{x}}_{I S O}+{ }^{R} \ddot{\boldsymbol{x}}_{\text {max }} * \tilde{t} .
$$

Here, ${ }^{R} \ddot{\boldsymbol{x}}_{\max }$ represents the robot deceleration which is depending on the robot type as well as the robot configuration, which has to be identified. The maximum allowed constant velocity is obtained by experiments with respect to the ISO/TS 15066 is ${ }^{R} \dot{\boldsymbol{x}}_{I S O}$. Finally, the improvement of the maximum velocity follows as ${ }^{R} \ddot{\boldsymbol{x}}_{\max } * \tilde{t}$.

To validate the developed algorithm for human arm motion estimation, the projected motions are compared to the human arm motion experiments from Sec. [III In Fig.9, the resulting projection is illustrated. The estimated motions are depicted by the black solid line, whereas the real measurements of the human arm motions are shown as blue solid line. The motion of the projection allows for a little higher velocity to guarantee a small safety threshold.

\section{Analysis of a typical collaborative scenario}

The motion projection enables the calculation of an estimated time a human hand needs to reach the robot path, i.e., the path of the end-effector. A sampling of projections in the human work space is depicted in Fig. 10 a), which illustrates that human arm motions can be estimated in every direction to the current robot path.

Fig. 10 b) illustrates a usual scenario of human-robot collaboration. The position $P_{0}$ describes the point entering the kinematic reachability of the human arm. Beyond the human arm work space, depicted as the black solid semicircle, the robot is allowed to move with maximum velocity. The maximum deceleration of the robot is given by ${ }^{R} \ddot{\boldsymbol{x}}_{\max }=$ $10 \mathrm{~m} / \mathrm{s}^{2}$ and the maximum velocity formalized by the ISO standards is given by ${ }^{R} \dot{\boldsymbol{x}}_{I S O}=0.1 \mathrm{~m} / \mathrm{s}$. The human arm needs a time of $\tilde{t}=110 \mathrm{~ms}$ to reach position $P_{0}$. With Eq. 8 a maximum velocity ${ }^{R} \dot{\boldsymbol{x}}_{\max }=1.2 \mathrm{~m} / \mathrm{s}$ is attained. At position $P_{2}$ the maximum robot velocity is obtained by ${ }^{R} \dot{\boldsymbol{x}}_{\max }=0.1 \mathrm{~ms}+10 \mathrm{~m} / \mathrm{s}^{2} \cdot 0.08 \mathrm{~s}=0.9 \mathrm{~m} / \mathrm{s}$. This is an enormous improvement of the robot performance and leads to an significant improvement of productivity in an industrial human-robot collaboration. To obtain still higher improvements, the follow-up paper "Safe and Efficient Human-Robot Collaboration Part II" will be extended with an integrated real-time path planner derived by [2] and an optimized path to the desired goal by minimizing the time according to $\min J=\int_{t_{0}}^{t_{f}} t^{2} d t$, considering the projected human arm motion.

\section{CONClusion}

In this paper, a new approach is presented towards a higher performance in industrial manufacturing with humanrobot collaboration. The paper contains a low-order, realtime capable human arm model to project a possible human arm motion pointing into the path of the robot. The main idea is to exploit the human arm dynamics to calculate the time required to attain the robot or the robot path, where a collision may occur. If this time span is large enough, it can be used to increase the velocity limit of the robot. The robot can move faster - without affecting the safety of the human operator - than prescribed by the ISO regulations, which are based on collision tests for peak force and peak pressure in transient and quasi-static contacts. Finally, the distance and time to reach the robotic path should be exploited to increase the velocity allowance of the robot. Only if a collision is possible, the robot has to decelerate and to reduce its velocity according to the limitations defined by the requirements of the ISO. The model has been validated with experimental data from a motion tracking system and real human arm motions. The difference to pure kinematic calculations is the correct description of real inertia effects in the dynamical simulation, which provide reliable and more 

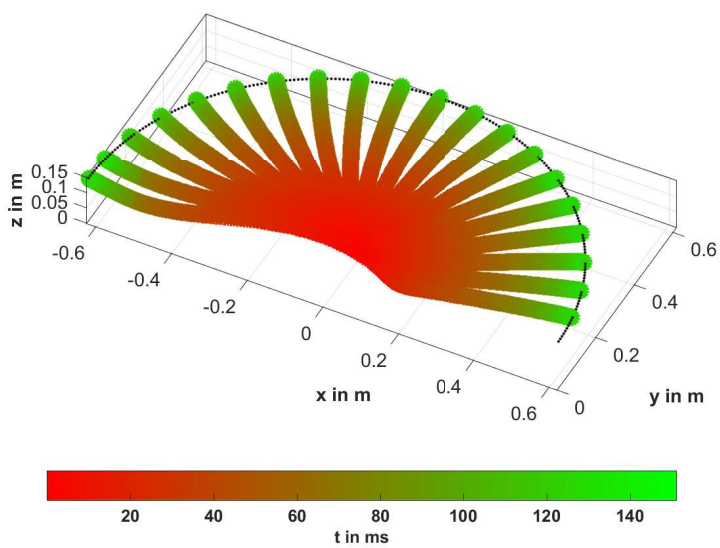

(a) Work space sampled simulation of human arm motions

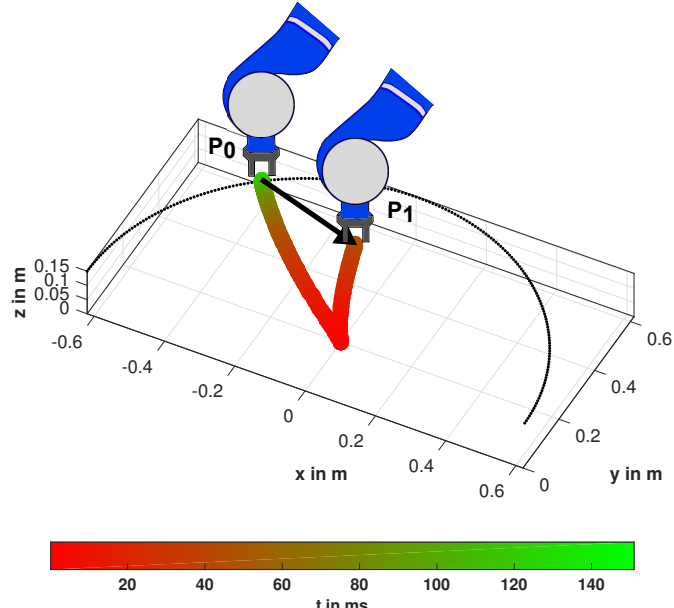

(b) Usual collaborative scenario

Fig. 10. Simulation of a sampled work space and analysis of a usual situation in human-robot collaboration

accurate results. Part of the future work is the a comparison of kinematic calculation and simulation, and implementing these information into real-time trajectory generators.

\section{ACKNOWLEDGEMENT}

We thank Freek Stulp and Alexander Dietrich for supporting this paper, Jörn Vogel for supporting this work with providing the VICON system, Thilo Wüsthoff for designing the figure of the human and the industrial schematic view. This work was partially funded by the Helmholtz Association in the project RACELab.

\section{REFERENCES}

[1] A. Albu-Schäffer, S. Haddadin, C. Ott, A. Stemmer, T. Wimb"ock, and G. Hirzinger, "The dlr lightweight robot - lightweight design and soft robotics control concepts for robots in human environments," Industrial Robot Journal, vol. 34, no. 5, pp. 376-385, 2007.

[2] R. Weitschat, J. Vogel, S. Lantermann, and H. Höppner, "Endeffector airbags to accelerate human-robot-collaboration," in IEEE International Conference on Robotics and Automation (ICRA), 2017.

[3] S. Haddadin, A. Albu-Schäffer, and G. Hirzinger, "Requirements for safe robots: Measurements, analysis and new insights," The International Journal of Robotics Research, vol. 28, no. 11-12, pp. 15071527, 2009.

[4] _ - "Safety evaluation of physical human-robot interaction via crash-testing." in Robotics: Science and Systems, vol. 3, 2007, pp. 217-224.

[5] L. Balan and G. M. Bone, "Real-time 3d collision avoidance method for safe human and robot coexistence," IEEE International Conference on Intelligent Robots and Systems (IROS), pp. 276 - 282, October 2006.

[6] O. Khatib, "Real-time obstacle avoidance for manipulators and mobile robots," in Autonomous robot vehicles. Springer, 1986, pp. 396-404.

[7] P. Vadakkepat, K. C. Tan, and W. Ming-Liang, "Evolutionary artificial potential fields and their application in real time robot path planning," in IEEE Proceedings of the Congress on Evolutionary Computation, vol. 1, 2000, pp. 256-263.

[8] H. S. Koppula and A. Saxena, "Anticipating human activities using object affordances for reactive robotic response," IEEE transactions on pattern analysis and machine intelligence, vol. 38, no. 1, pp. 1429, 2016.

[9] J. Mainprice and D. Berenson, "Human-robot collaborative manipulation planning using early prediction of human motion," in IEEE/RSJ International Conference on Intelligent Robots and Systems (IROS), 2013, pp. 299-306.
[10] S. Hamasaki, Y. Tamura, A. Yamashita, and H. Asama, "Prediction of human's movement for collision avoidance of mobile robot," in IEEE International Conference on Robotics and Biomimetics (ROBIO), 2011, pp. 1633-1638.

[11] D. Kulić and E. Croft, "Pre-collision safety strategies for human-robot interaction," Autonomous Robots, vol. 22, no. 2, pp. 149-164, 2007.

[12] H. Noguchi, T. Yamada, T. Mori, and T. Sato, "Mobile robot path planning using human prediction model based on massive trajectories," in IEEE International Conference on Networked Sensing Systems (INSS), 2012, pp. 1-7.

[13] G. Ferrer and A. Sanfeliu, "Behavior estimation for a complete framework for human motion prediction in crowded environments," in IEEE International Conference on Robotics and Automation (ICRA), 2014, pp. 5940-5945.

[14] A. Babiarz, A. Czornik, J. Klamka, M. Niezabitowski, and R. Zawiski, "The mathematical model of the human arm as a switched linear system," in IEEE 19th International Conference On Methods and Models in Automation and Robotics (MMAR), 2014, pp. 508-513.

[15] H. J. Nagarsheth, P. V. Savsani, and M. A. Patel, "Modeling and dynamics of human arm," in IEEE International Conference on Automation Science and Engineering (CASE), 2008, pp. 924-928.

[16] E. K. Chadwick, D. Blana, R. F. Kirsch, and A. J. Van Den Bogert, "Real-time simulation of three-dimensional shoulder girdle and arm dynamics," IEEE Transactions on Biomedical Engineering, vol. 61, no. 7, pp. 1947-1956, 2014.

[17] R. Dumas, L. Cheze, and J. Verriest, "Adjustments to mcconville et al. and young et al. body segment inertial parameters," Journal of Biomechanics, vol. 40, no. 7, pp. 1651-1652, 2007.

[18] C. Simonidis, S. Gaertner, and M. Do, "Spezifikationen zu den ganzkoerpermenschmodellen im sfb 588," Technical report, Universitaets Verlag Karlsruhe, Tech. Rep., 2009.

[19] A. Dietrich, T. Wimböck, and A. Albu-Schäffer, "Dynamic wholebody mobile manipulation with a torque controlled humanoid robot via impedance control laws," in IEEE/RSJ International Conference on Intelligent Robots and Systems (IROS), 2011, pp. 3199-3206.

[20] E. D. Fasse and J. F. Broenink, "A spatial impedance controller for robotic manipulation," IEEE Transactions on Robotics and Automation, vol. 13, no. 4, pp. 546-556, 1997.

[21] C. W. I1 and L. Leifer, "Applications of damped least-squares methods to resolved-rate and resolved acceleration control of manipulators," in J. Dyn. Syst., Meas. Contr, vol. 110, 1988, pp. 31-38. 